\title{
Genetic evolution and implications of the mitochondrial genomes of two newly identified Taenia spp. in rodents from Qinghai-Tibet Plateau
}

\author{
Yao-Dong $\mathrm{Wu}^{1}$, Li Li ${ }^{1}$, Yan-Lei Fan ${ }^{1}$, Xing-Wei Ni ${ }^{1}$, John Ohiolei ${ }^{1}$, Wenhui Li ${ }^{2}$, Jian-Qiu \\ $\mathrm{Li}^{1}$, Nianzhang Zhang ${ }^{2}$, Baoquan $\mathrm{Fu}^{3}$, Hongbin Yan ${ }^{1}$, and Wanzhong $\mathrm{Jia}^{1}$ \\ ${ }^{1}$ Lanzhou Veterinary Research Institute \\ ${ }^{2}$ Affiliation not available \\ ${ }^{3}$ Lanzhou Veterinary Research Institute, Chinese Academy of Agricultural Sciences
}

November 5, 2020

\begin{abstract}
The larva of Taeniidae species can infect a wide range of mammals, causing major public health and food safety hazards worldwide. The Qinghai-Tibet Plateau (QTP), a biodiversity hotspot, is home to many species of rodents, which act as the critical intermediate hosts of many Taeniidae species. In this study, we identified two new larvae of Taenia spp., named as T. caixuepengi and T. tianguangfui, collected from the plateau pika (Ochotona curzoniae) and the Qinghai vole (Neodon fuscus), respectively in QTP, and their mitochondrial genomes were sequenced and annotated. Phylogenetic trees based on the mitochondrial genome showed that T. caixuepengi has the closest genetic relationship with T. pisiformis, while T. tianguangfui was contained in a monophyletic group with T. crassiceps, T. twitchelli and T. martis. Biogeographic scenarios analysis based on split time speculated that the speciation of T. caixuepengi ( $\sim 5.49 \mathrm{Mya})$ is due to host switching caused by the evolution of its intermediate host. Although the reason for T. tianguangfui ( $\sim 13.11 \mathrm{Mya})$ speciation is not clear, the analysis suggests that it should be infective to a variety of other rodents following the evolutionary divergence time of its intermediate host and the range of intermediate hosts of its genetically close species. This study confirms the species diversity of Taeniidae in the QTP, and speculates that the uplift of the QTP has not only a profound impact on the biodiversity of plants and animals, but also that of parasites.
\end{abstract}

Genetic evolution and implications of the mitochondrial genomes of two newly identified Taenia spp. in rodents from Qinghai-Tibet Plateau

Authors: Yao-Dong $\mathrm{Wu}^{1}$, $\mathrm{Li} \mathrm{Li}^{1}$, Yan-Lei Fan ${ }^{1,2}$, Xing-Wei $\mathrm{Ni}^{1,3}$, John Asekhaen Ohiolei ${ }^{1}$, Wen-Hui $\mathrm{Li}^{1}$, Jian-Qiu Li ${ }^{1}$, Nian-Zhang Zhang ${ }^{1}$, Bao-Quan $\mathrm{Fu}^{1}$, Hong-Bin $\mathrm{Yan}^{1 *}$, Wan-Zhong Jia ${ }^{1,4 *}$

\section{Affiliations}

1. State Key Laboratory of Veterinary Etiological Biology/ National Professional Laboratory for Animal Echinococcosis / Key Laboratory of Veterinary Parasitology of Gansu Province/ Lanzhou Veterinary Research Institute, CAAS, Lanzhou 730046, P. R. China.

2. School of Pharmaceutical Sciences, Tsinghua University, Beijing 100084, China.

3. Animal Disease Prevention and Control Center of Guizhou Province, Guiyang 550008, P. R. China.

4. Jiangsu Co-innovation Center for Prevention and Control of Important Animal Infectious Disease, Yangzhou 225009, P. R. China. 


\title{
List of authors and emails:
}

YD Wu: 510510.good@163.com

L Li:lili03@caas.cn

YL Fan: fanyl69@163.com

XW Ni:961679487@qq.com

JA Ohiolei:asekhaenj@gmail.com

WH Li:liwenhui@caas.cn

JQ Li:709404514@qq.com

NZ Zhang:nianzhang919@163.com

BQ Fu: fubaoquan@caas.cn

* Corresponding authors: WZ Jia:jiawanzhong@caas.cn; HB Yan:yanhongbin@caas.cn

\begin{abstract}
The larva of Taeniidae species can infect a wide range of mammals, causing major public health and food safety hazards worldwide. The Qinghai-Tibet Plateau (QTP), a biodiversity hotspot, is home to many species of rodents, which act as the critical intermediate hosts of many Taeniidae species. In this study, we identified two new larvae of Taenia spp., named as T. caixuepengi and T. tianguangfui, collected from the plateau pika (Ochotona curzoniae) and the Qinghai vole (Neodon fuscus ), respectively in QTP, and their mitochondrial genomes were sequenced and annotated. Phylogenetic trees based on the mitochondrial genome showed that $T$. caixuepengi has the closest genetic relationship with T. pisiformis, while T. tianguangfui was contained in a monophyletic group with T. crassiceps, T. twitchelli and T. martis . Biogeographic scenarios analysis based on split time speculated that the speciation of $T$. caixuepengi( $\sim 5.49$ Mya) is due to host switching caused by the evolution of its intermediate host. Although the reason for T. tianguangfui ( 13.11 Mya) speciation is not clear, the analysis suggests that it should be infective to a variety of other rodents following the evolutionary divergence time of its intermediate host and the range of intermediate hosts of its genetically close species. This study confirms the species diversity of Taeniidae in the QTP, and speculates that the uplift of the QTP has not only a profound impact on the biodiversity of plants and animals, but also that of parasites.
\end{abstract}

Keywords: Taenia spp., mtDNA, Qinghai-Tibet Plateau, Phylogeny, Divergence time

Running head: Two newly identified Taenia spp. in China

\section{Introduction}

The most recent molecular phylogenetic analysis has suggested that the family Taeniidae (Eucestoda: Cyclophyllidae) should be composed of four genera:Taenia , Echinococcus , Hydatigera and Versteria (Nakao et al., 2013). Among them, Taenia andEchinococcus species pose a serious public health threat to humans and animals globally. Terrestrial mammals are crucial to the life cycle of taeniids. Adult tapeworm of most taeniid species parasitizes the intestine of carnivores, while their eggs infect the intermediate host, usually herbivores and omnivores as well as humans, through fecal-oral route, and develop into larvae, which cause severe health effects (Deplazes et al., 2019; Jia et al., 2012; Lymbery, 2017; Nakao et al., 2013).

Before Nakao's new classification recommendation, Taeniidae was generally supposed to consist of two valid genera, Taenia andEchinococcus, and the genus Taenia (Linnaeus, 1758) was demarcated approximately into 42 valid species and three subspecies by adult and metacestode morphology (Hoberg, 2006; Hoberg et al., 2000; Nakao et al., 2013). As for Echinococcus, a total of 16 species and 13 subspecies were described based on morphology before the widespread application of molecular genetic methods, but most of these taxa were subsequently regard as invalid (Lymbery, 2017). It is difficult to distinguish taeniid species according 
to their morphological characteristics at different stages of their life cycle, even by specialists (Flisser et al., 2005; Jia et al., 2012; Mathis \& Deplazes, 2006). Sometimes, morphological characteristics are substantially influenced by the different intermediate host origins (Lymbery, 1998).

Mitochondrial (mt) DNA sequence has been recognized among the most suitable molecular markers of molecular ecology, population genetics, evolutionary biology and biological differentiation due to its high mutation rate and maternal inheritance (Jia et al., 2012; Hajibabaei et al., 2007; Hebert \& Gregory, 2005; Will et al., 2005). In the last two decades, comparative analyses of taeniid mtDNAs have been increasingly applied to phylogenetic studies, from estimating the evolutionary status for new species identification and even species reclassification, to investigating the phylogeography and genetic diversity for tracing the evolutionary origins of related and identical species (Kinkar et al., 2018; Nakao et al., 2007; Nakao et al., 2013; Terefe et al., 2014; Xiao et al., 2005). Among the taeniid family, mt genomes of 36 species and genotypes have been sequenced and are available on GenBank (https://www.ncbi.nlm.nih.gov/genbank/ ), providing valuable data support for phylogenetic studies of Taeniidae.

The shrinkage and fragmentation of wildlife habitats due to human activities can lead to increased contact between humans or livestock and wildlife, which potentially increases the risk of transmission of natural focal disease (Suzán et al., 2008). Rodents, the largest ( $\sim 43 \%$ of all mammal species) and most widely distributed group of mammals, act as major vectors of human and domestic animal diseases (Singla et al., 2008; Wu et al., 2018). The Qinghai-Tibet Plateau (QTP), one of the biodiversity hotspots on earth, is habitat to a rich diversity of wild rodent species (Zhou \& Ma, 2002), as well as many rodent-eating carnivores (Smith et al., 2019), creating the conditions for various taeniid species to complete their life cycles. The high altitude geographic isolation combined with the geological complexity of the QTP increases the opportunities for genetic variation and speciation, leading to the continuous discovery of new species within rodents and Taeniidae (Dahal et al., 2017; Xiao et al., 2005). However, few studies have involved the population structure and biodiversity of taeniid species in QTP, except for Echinococcus .

As endangered or protected carnivores are difficult to sample, we collected metacestode samples of rodents to investigate the biodiversity and distribution of taeniid species in QTP. In this study, two new mt genomes of the metacestode samples were firstly sequenced and annotated. Through the phylogenetic analysis of $\mathrm{mt}$ genomes with species in the four different genera of taeniids, the validity of these two new Taenia spp., named as T. caixuepengiand T. tianguangfui larvae, were confirmed and their phylogenetic relationship and evolutionary origin were analyzed.

\section{Materials and methods}

\subsection{Parasite Materials}

Plateau pikas (Ochotona curzoniae) and Qinghai voles (Neodon fuscus) were trapped in Darlag county $\left(33^{\circ} 43^{`} \mathrm{~N} ; 99^{\circ} 38^{`} \mathrm{E}\right.$; altitude at $\left.4068 \mathrm{~m}\right)$ and Jiuzhi county $\left(33^{\circ} 19^{`} \mathrm{~N} ; 100^{\circ} 32^{\circ} \mathrm{E}\right.$; altitude at $\left.3832 \mathrm{~m}\right)$ of Qinghai province, the People's Republic of China in July 2013. Following ethical approval, all trapped pikas and voles were dissected regarding the enterocoelia, chest and cranial cavities. Many banded cysticerci were collected in the enterocoelia of Plateau pika (Supplementary Fig. S1A and B) and numerous lenticular cysticerci were collected in the enterocoelia and chest of Qinghai vole (Supplementary Fig. S1C and D). After detaching the lesions, the cysticerci were put into $75 \%$ (v/v) ethanol for molecular identification.

\subsection{DNA isolation, amplification and sequencing}

Cysticercus DNA was extracted using a commercial kit as instructed by the manufacturer (Blood and Tissue Kit, Qiagen, Germany). The mt genomes of Taenia spp., whose intermediate hosts include rodents, downloaded from GenBank (Supplementary Table S2) were aligned by using MEGA 7.0. Nine overlapping primers targeting the complete mt genome were designed using Oligo 6.0 at relatively conserve regions observed on alignment of the mt genome sequences. The primer sequences (Supplementary Table S1) were synthesized by Genewiz Biotech (Beijing, China). A standard $50 \mu \mathrm{l} \mathrm{PCR} \mathrm{protocol} \mathrm{was} \mathrm{used} \mathrm{to} \mathrm{amplify} \mathrm{the}$ mtDNA fragments. PCR products were purified directly from an agarose gel (1\%) using an Axy Prep DNA 
Gel Extraction Kit (AXVGEN, USA) and then sent to the company Genewiz Biotech for sequencing.

\subsection{Mitochondrial genome annotation}

These two mtDNAs were assembled manually, and annotated preliminarily by Geseq (https://chlorobox.mpimp-golm.mpg.de/geseq.html) with the reference of related species, T. pisiformis and T. crassiceps, identified by the cox 1 gene alignment of Neighbor-Joining method in MEGA 5.1 (data not show). Putative tRNA genes were identified using ARWEN (http://130.235.46.10/ARWEN/) (Laslett \& Canbäck, 2008). The positions of their open reading frames and rRNA genes were also further checked and modified based on alignment with the $\mathrm{mt}$ genomes annotation of T. pisiformis and T. crassiceps , respectively. SnapGene (v3.2.1) was used to translate the amino acid sequence of the protein-coding genes with genetic code of the Echinoderm, Flatworm Mitochondrial and map the annular diagram of the mt genomes.

\subsection{Phylogenetic analyses}

To determine the phylogenetic status of these two Taenia spp., the phylogenetic trees were constructed using Bayesian methods in MrBayes v3 with the tandem DNA sequences and amino acid sequences of 12 encoding genes in their mt genomes and other 32 taeniid mt genome sequences downloaded from GenBank, while the sequences of Schistosoma japonicum was used as outgroup (Supplementary Table S2). For the amino acid data set, the mixed model was applied (prset aamodelpr $=$ mixed); two chains (temp $=0.2$ ) were run for $3,000,000$ generations and sampled every 1,000 generations. For the nucleotide data set, Modeltest 3.7 maxX (Posada and Crandall, 1998) was used to estimate a suitable model for nucleotide substitution; this was equivalent to $\mathrm{GTR}+\mathrm{I}+\mathrm{G}$ and settings were $\mathrm{nst}=6$, rates $=$ invgamma, ngammacat $=4$. Four chains $($ temp $=0.2$ ) were run for 1,000,000 generations and sampled every 1,000 generations. The first $25 \%$ of trees were omitted as burn-in and the remaining trees were used to calculate Bayesian posterior probabilities. The best Bayesian tree was then compiled and processed by FigTree.v1.4.4.

\subsection{Divergence times analysis}

The phylogenetic trees were used as a reference for species selection in divergence times analysis. Echinococcus multilocularis and $E$. shiquicus were also selected because the parasitism of their larvae is also found in Plateau pika (O. curzoniae) (Wang et al., 2018), besides E. multilocularis is the sister species of E. shiquicus (Lymbery, 2017). Divergence times were calculated from the concatenated CDS alignment of the 12 mitochondrial protein-coding genes by BEAST2 (v2.6.2). The Strict Clock model was chosen to ignore the rate differences between the branches in the mode. The gamma category count was set to 4, and HKY substitution model was selected with the empirical setting from the frequencies in site model. Other settings, such as substitution rate and shape, in the site model were evaluated in the analysis. The calibrated Yule model was used as the tree prior. Time calibration was calibrated with the previously estimated date between T. saginata and T. asiatica ( ${ }^{1.14}$ Mya) (Michelet \& Dauga, 2012; Wang et al., 2016). Samples from the posterior were drawn every 1,000 steps over a total of 10,000,000 steps per MCMC run. Other options were run on their default values. The convergence of likelihood values was determined by Tracer (v1.7.1). Trees were annotated by TreeAnnotator (v2.1.2) using maximum clade credibility tree and median heights settings with $50 \%$ burn-in. The evolutionary divergence time of the intermediate host, Qinghai vole ( $N$. fuscus ), was also calculated with the concatenated CDS alignment of $13 \mathrm{mt}$ protein-coding genes of the rodents, and the species involved were selected from our previous report (Li et al., 2019) (Supplementary Table S2). The time calibration was based on the divergence time of Mus and Rattus (11-13 Mya) (Wang et al., 2020), and other parameters were the same as above.

\section{Results}

\subsection{General features of the mitochondrial genome of two parasites}

The complete mt genomes of the cysticerci collected from Qinghai vole and Plateau pika, named as T. caixuepengi larva (GenBank ID: MT882036) and T. tianguangfui larva (GenBank ID: MT882037), were $13,747 \mathrm{bp}$ and $13,522 \mathrm{bp}$ in length, respectively. Both of them contain 2 rRNA genes (the small [ rrn S] and 
large $[r r n \mathrm{~L}]$ subunits of rRNA), 12 protein-encoding genes (atp 6, cyt b, nad 4L, cox 1-3 and nad 1-6) and 22 tRNA genes, but lack atp 8 gene, which are typical of cestode mt genomes (Fig. 1). The inferred gene boundaries and their lengths are shown in Table 1.

In accordance with other mtDNAs of flatworms sequenced to date (Liu et al., 2011; Jia et al., 2010), the nucleotide compositions are mostly biased toward T, while least favored toward C. AT-richness of mtDNAs in T. caixuepengi and T. tianguangfui are $71.96 \%(45.00 \% \mathrm{~T}, 26.97 \% \mathrm{~A}, 19.17 \% \mathrm{G}, 8.87 \% \mathrm{C})$ and $73.48 \%$ (46.35\% T, $27.13 \%$ A, $18.61 \%$ G, $7.91 \%$ C), respectively.

Flatworms use an unusual mt code to exert protein translation (Nakao et al., 2000; Telford et al., 2000). GTG was used as an alternative initiation codon in cox 3 and nad 3 genes of $T$. caixuepengi and nad 5 gene of T. tianguangfui . Furthermore, the codon ATT was inferred as a more unusual start codon of atp 6 gene in T. caixuepengi. The termination codon was mostly TAA, and the ending codon TGA was deprecated (Table 1).

\subsection{Phylogenetic relationships}

Phylogenies inferred from both tandem amino acid sequences and DNA sequences of the $12 \mathrm{mt}$ proteinencoding genes demonstrated that T. caixuepengi in a monophyletic group with T. pisiformis and T. laticollis , with the closest genetic relative being T. pisiformis ; T. tianguangfui was also found in a monophyletic group with T. crassiceps, T. twitchelli and T. martis, and has the far genetic relative with T. caixuepengi(Fig. 2).

\subsection{Divergence times analysis}

The divergence time analysis based on mitochondrial protein-coding genes suggested that $T$. saginata and T. asiatica should diverge at 1.10 Mya (0.80-1.41, $95 \%$ highest probability density) in the early Pleistocene period, which is consistent with the previous reports based on genomic genes (Michelet and Dauga, 2012; Wang et al., 2016); T. caixuepengi should diverge from T. pisiformis 5.49 Mya (3.87-7.19, 95\% highest probability density) in the initial Pliocene period, which is close to the divergence time between E. shiquicus and E. multilocularis (4.12 Mya, 2.81-5.32, 95\% highest probability density); T. tianguangfui on the other hand, originated 13.11 Mya (9.36-17.18, 95\% highest probability density) in the middle Miocene period, which was earlier than the differentiation of its intermediate host, N. fuscus (4.98 Mya, 4.08-5.90, 95\% highest probability density) (Fig. 3, Supplementary Fig. S2).

\section{Discussion}

The discovery of these two new parasites, T. caixuepengi and T. tianguangfui, highlights the species diversity of the family Taeniide, and further proved the true biodiversity characteristic of the QTP. Given the lack of human intervention and the rich diversity of wild host species, the present understanding of the species diversity within this family in QTP is apparently just a tip of the iceberg. This is not surprising, given the appreciable cryptic diversity so far uncovered within the taeniid family in Africa and northern latitudes (Lavikainen et al., 2011; Lavikainen et al., 2013; Terefe et al., 2014).

Here the whole mt genomes of both new species were sequenced, and were clearly different from all available Taenia mt genome sequences, verifying the validity of their species status. Their mt genomes were similar as those of other sequenced tapeworms with respect to length, nucleotide bias, and their tRNA, rRNA and protein-encoding genes composition (Fig. 1) (Jeon et al., 2005; Le et al., 2000; Nakao et al., 2003). Furthermore, the codon ATT was inferred as a more unusual start codon for the atp 6 gene of $T$. caixuepengi (Table 1), which is a common start codon used by Caenorhabditis elegans and Ascaris suum (Okimoto et al., 1990).

T. caixuepengi larva is so far undetected in other animals, except plateau pika (O. curzoniae), meanwhile, no other cysticerci have been found in plateau pika hitherto. Lagomorpha is the intermediate host of T. pisiformis and T. laticollis(Hallal-Calleros et al., 2016; Valdmann et al., 2004). Although similar in appearance and size to the vole, the plateau pika belongs to the Lagomorpha (Smith et al., 2019). The close phylogenetic 
relatedness of these three Taenia species (Fig. 2) is further highlighted by their high preference for lagomorphs as an intermediate host. Based on the divergence time and phylogeographic analyses, the extent pikas (genus Ochotona ) originated on the QTP in the middle Miocene, 14 Mya (Wang et al., 2020). However, the rapid speciation of many Ochotona species, including $O$. curzoniae, occurred during the late Miocene and early Pliocene period (Wang et al., 2020), which almost coincided with the rapid uplift of the QTP (An et al., 2006; Li et al., 2007; Shi et al., 2015). Coincidentally, the evolutionary divergence time analysis in this study also suggests that both T. caixuepengi and E. shiquicus had evolved in the early Pliocene epoch, about 5.49 Mya and 4.12 Mya, respectively (Fig. 3). These almost synchronous events may not have happened by chance. Large-scale diversification of species is often provoked by abiotic factors, such as changes in the living environment and food supply (Benton, 2009). The uplift of the QTP from south to north provided climatic opportunities and food supply for the diversification of cold temperature-preferring pikas but led to the extinction of other warm temperature-preferring rodents (Wang et al., 2020).

For most free-living organisms, speciation is usually the result of genetic drift or adaptive differentiation between geographically separate populations (Turelli et al., 2001). For parasites, however, it has long been thought that sympatric speciation of parasites is common, mediated by ecological isolation caused by host switching within the same geographic region (de Meeûs et al., 1998; Huyse et al., 2005; Paul, 2002). Therefore, we speculate that $T$. pisiformi $\mathrm{s}$ in the QTP (Li et al., 2013) may share a common ancestor with $T$. caixuepengi ; the split of the pika population caused the ecological isolation between their ancestral populations, which further resulted in the lack of gene flow between them due to intermediate host switching, and the eventual formation of two different species. It can also be speculated that the differentiation pattern between E. shiquicus and E. multilocularis is similar as that of T. caixuepengi and T. pisiformis .

Our evolutionary divergence time analysis suggests that the speciation of $T$. tianguangfui occurred in the middle Miocene period ( ${ }^{\sim} 13.11$ Mya) (Fig. 3) when the QTP was undergoing a slow uplift period (An et al., 2006). The timing of the divergence of $N$. fuscus evolved from 4.98 Mya (Supplementary Fig. S2), which also coincided with the rapid uplift of the QTP (An et al., 2006; Li et al., 2007; Shi et al., 2015). As the species spread in the QTP and Himalaya (Pradhan et al., 2019), the evolutionary origin of theNeodon spp., like the plateau pika, may well be due to changes in climate and food supply caused by the uplift of the QTP and Himalaya. The speciation of T. tianguangfui was earlier than that of its intermediate host, indicating that T. tianguangfui did not differentiate into a new Taenia species in order to adapt to the intermediate host, rather, it suggests that $T$. tianguangfui larva might not be limited to $N$. fuscus. Taenia crassiceps and $T$. martis have similar intermediate hosts range, infecting a variety of rodents, even humans and other primates (Deplazes et al., 2019). Given the close relationship between T. tianguangfui, T. crassiceps and T. martis, it also cannot be excluded that $T$. tianguangfui may be infective to a variety of rodents other than $N$. fuscus , as well as humans and other primates. So far, a clear understanding of their evolutionary origin from these clues is elusive, thus, more data and investigation are needed to provide further insight.

Adult worms of the T. tianguangfui and T. caixuepengi have not yet been collected due to the difficulty in sampling endangered or protected carnivores. Plateau pikas and voles are the primary food source for wild canids across the QTP. Tibetan foxes are the obligate predator of plateau pikas, as their remains (plateau pikas) are often encountered in $99 \%$ of their feces (Smith et al., 2019). Wild canids, especially the red fox and the Tibetan fox, may well be important definitive hosts for T. tianguangfui and T. caixuepengi .

Adult or larval samples of tapeworm are easily damaged in the process of collection, freeze-thaw and processing, and the morphological features are mostly unidentifiable (Lavikainen et al., 2013). While mt genome data alone may not fully answer the scientific questions surrounding their evolutionary origins, it is the most cost-effective and accurate method. Recently, although laborious and costly, there have been an increasing whole genome sequencing and analyses for many tapeworm species. This kind of investigation, not only is it important to provide insights into their host adaptation and switching, evolution mechanisms through gene groups amplification, hosts-parasites interaction, immune regulation and nutrition, it also provides urgently needed resources for the identification of drug target and diagnostic molecular markers (International Helminth Genomes Consortium, et al., 2019; Wang et al., 2016). In the future, a lot of genomic data will be 
needed to study this fascinating group.

\section{Conclusion}

Finally, the mitochondrial genome sequence data adequately confirm the validity of the two new Taenia species named T. caixuepengi and T. tianguangfui, we have previously reported. The phylogenetic trees and divergence times analysis suggest that $T$. caixuepengi evolve from its closest relative, T. pisiformis, in the initial Pliocene period ( $\sim 5.49$ Mya), due to the intermediate host switching caused by the rapid uplift of the QTP; T. tianguangfui be probably parasitic in a wide variety of rodents, and share a common ancestor with T. crassiceps, T. twitchelli and T. martis, splitting in middle Miocene period ( ${ }^{13.11}$ Mya).

\section{Appendex A. Supplementary material}

Table S1. The primers for amplifying the complete mitochondrial genomes of cysticercus. Table S2. GenBank accession numbers of mitochondrial genome sequences used for phylogenetic analyses and divergence times analyses in this study. Fig. S1. The larvae of Taenia caixuepengi and T. tianguangfui were found in Plateau pikas (Ochotona curzoniae) and Qinghai voles (Neodon fuscus), respectively. Fig. S2. Divergence times construction for Neodon fuscus based on the concatenated CDS alignments of mitochondrial 13 protein-encoding genes.

\section{Acknowledgements}

This work was funded by the National Key Research and Development Plan (2018YFC1602504; 2017YFD0501301), Central Public-Interest Scientific Institution Basal Research Fund (1610312020016), and Cultivation of Achievements of State Key Laboratory of Veterinary Etiological Biology (SKLVEB2020CGPY01).

\section{Conflicts of Interest}

The authors declare no conflict of interest.

\section{Ethics Statement}

All animals were handled in strict accordance with good animal practice according to the Animal Ethics Procedures and Guidelines of the People's Republic of China, and the study was approved by the Animal Ethics Committee of Lanzhou Veterinary Research Institute, Chinese Academy of Agricultural Sciences (No. LVRIAEC2012-007).

\section{Data availability statement}

The data of mt genomes phylogenetic analysis and design of primers used to support the findings of this study are included within the supporting information. The data of mt genomes accession numbers used to support the findings of this study are included within the website of NCBI.

\section{Reference}

An, Z.S., Zhang, P.Z., Wang, E.Q., Wang, S.M., Qiang, X.K., Li, L., ... Ai, L. (2006). Changes of the monsoon-arid environment in China and growth of the Tibetan Plateau since the Miocene. Quaternary Sciences , 26, 678-693. (in Chinese).

Benton, M.J. (2009). The Red Queen and the Court Jester: species diversity and the role of biotic and abiotic factors through time. Science, 323, 728-732. https://doi.org/10.1126/science.1157719.

Dahal, N., Lissovsky, A.A., Lin, Z., Solari, K., Hadly, E.A., Zhan, X., \& Ramakrishnan, U. (2017). Genetics, morphology and ecology reveal a cryptic pika lineage in the Sikkim Himalaya. Molecular Phylogenetics and Evolution, 106, 55-60.https://doi.org/10.1016/j.ympev.2016.09.015.

de Meeus, T., Michalakis, Y., \& Renaud, F. (1998). Santa rosalia revisited: or why are there so many kinds of parasites in; the garden of earthly delights'?.Parasitology Today (Personal ed.) , 14, 1013.https://doi.org/10.1016/s0169-4758(97)01163-0. 
Deplazes, P., Eichenberger, R.M., \& Grimm, F. (2019). Wildlife-transmitted Taenia and Versteria cysticercosis and coenurosis in humans and other primates. International Journal for Parasitology. Parasites and Wildlife , 9, 342-358.https://doi.org/10.1016/j.ijppaw.2019.03.013.

Flisser, A., Correa, D., Avilla, G., \& Marvilla, P. (2005). Biology of Taenia solium ,Taenia saginata and Taenia saginata asiatica. In: Murrell, K.D. Paris (Ed.), WHO/FAO/OIE Guidelines for the Surveillance, Prevention and Control of Taeniosis/Cysticercosis. OIE Press , pp, 14-22.

Hajibabaei, M., Singer, G.A., Hebert, P.D., \& Hickey, D.A. (2007). DNA barcoding: how it complements taxonomy, molecular phylogenetics and population genetics. Trends in Genetics: TIG, 23, 167172.https://doi.org/10.1016/j.tig.2007.02.001.

Hallal-Calleros, C., Morales-Montor, J., Orihuela-Trujillo, A., Togno-Peirce, C., Murcia-Mejia, C., Bielli, A., ... Flores-Perez, F.I. (2016). Taenia pisiformis cysticercosis induces decreased prolificacy and increased progesterone levels in rabbits. Veterinary Parasitology , 229, 5053.https://doi.org/10.1016/j.vetpar.2016.09.015.

Hebert, P.D., \& Gregory, T.R. (2005). The promise of DNA barcoding for taxonomy. Systematic Biology, 54, 852-859.https://doi.org/10.1080/10635150500354886.

Hoberg, E. P., Jones, A., Rausch, R. L., Eom, K. S., \& Gardner, S. L. (2000). A phylogenetic hypothesis for species of the genus Taenia(Eucestoda: Taeniidae). The Journal of Parasitology , 86, 89-98. https://doi.org/10.1645/0022-3395(2000)086[0089:APHFSO]2.0.CO;2.

Hoberg E. P. (2006). Phylogeny of Taenia : Species definitions and origins of human parasites. Parasitology International , 55, S23-S30. https://doi.org/10.1016/j.parint.2005.11.049.

Huyse, T., Poulin, R., \& Theron, A. (2005). Speciation in parasites: a population genetics approach. Trends in Parasitology , 21, 469-475.https://doi.org/10.1016/j.pt.2005.08.009.

International Helminth Genomes Consortium. (2019). Comparative genomics of the major parasitic worms. Nature Genetics , 51, 163-174.https://doi.org/10.1038/s41588-018-0262-1.

Jeon, H.K., Kim, K.H., \& Eom, K.S. (2007). Complete sequence of the mitochondrial genome of Taenia saginata : comparison with T. solium and T. asiatica . Parasitology International , 56, 243246.https://doi.org/10.1016/j.parint.2007.04.001.

Jeon, H.K., Lee, K.H., Kim, K.H., Hwang, U.W., \& Eom, K.S. (2005). Complete sequence and structure of the mitochondrial genome of the human tapeworm, Taenia asiatica (Platyhelminthes; Cestoda).Parasitology , 130, 717-726.https://doi.org/10.1017/s0031182004007164.

Jia, W.Z., Yan, H.B., Guo, A.J., Zhu, X.Q., Wang, Y.C., Shi, W.G., ., Cai, X.P. (2010). Complete mitochondrial genomes of Taenia multiceps , T. hydatigena and T. pisiformis : additional molecular markers for a tapeworm genus of human and animal health significance. BMC Genomics , 11, 447.https://doi.org/10.1186/1471-2164-11-447.

Jia, W., Yan, H., Lou, Z., Ni, X., Dyachenko, V., Li, H., \& Littlewood, D.T. (2012). Mitochondrial genes and genomes support a cryptic species of tapeworm within Taenia taeniaeformis . Acta Tropica , 123, 154-163. https://doi.org/10.1016/j.actatropica.2012.04.006.

Kinkar, L., Laurimae, T., Acosta-Jamett, G., Andresiuk, V., Balkaya, I., Casulli, A., ... Saarma, U. (2018). Global phylogeography and genetic diversity of the zoonotic tapeworm Echinococcus granulosus sensu stricto genotype G1. International Journal for Parasitology , 48, 729742.https://doi.org/10.1016/j.ijpara.2018.03.006.

Laslett, D., \& Canback, B. (2008). ARWEN: a program to detect tRNA genes in metazoan mitochondrial nucleotide sequences. Bioinformatics (Oxford, England) , 24, 172175.https://doi.org/10.1093/bioinformatics/btm573 . 
Lavikainen, A., Haukisalmi, V., Deksne, G., Holmala, K., Lejeune, M., Isomursu, M., .. Sukura, A. (2013). Molecular identification of Taenia spp. in the Eurasian lynx (Lynx lynx ) from Finland.Parasitology , 140, 653-662.https://doi.org/10.1017/S0031182012002120.

Lavikainen, A., Laaksonen, S., Beckmen, K., Oksanen, A., Isomursu, M., \& Meri, S. (2011). Molecular identification of Taenia spp. in wolves (Canis lupus), brown bears (Ursus arctos) and cervids from North Europe and Alaska. Parasitology International ,60, 289-295.https://doi.org/10.1016/j.parint.2011.04.004.

Le, T.H., Blair, D., Agatsuma, T., Humair, P.F., Campbell, N.J., Iwagami, M., .. McManus, D.P. (2000). Phylogenies inferred from mitochondrial gene orders-a cautionary tale from the parasitic flatworms. Molecular Biology and Evolution , 17, 1123-1125.https://doi.org/10.1093/oxfordjournals.molbev.a026393.

Li, D.P., Zhao, Y., Hu, J.M., Wan, J.L., Li, X.L., Zhou, X.K., ... Pei, J.L. (2007). Fission track thermochronologic constraints on plateau surface and geomorphic relief formation in the northwestern margin of the Tibetan Plateau. Acta Petrologica Sinica, 23, 900-910. (in Chinese)

Li, J.Q., Li, L., Fu, B.Q., Yan, H.B., \& Jia, W.Z. (2019). Complete mitochondrial genomes confirm the generic placement of the plateau vole,Neodon fuscus . Bioscience Reports , 39, BSR20182349.https://doi.org/10.1042/BSR20182349.

Li, W., Guo, Z., Duo, H., Fu, Y., Peng, M., Shen, X., ... Nonaka, N. (2013). Survey on helminths in the small intestine of wild foxes in Qinghai, China. The Journal of Veterinary Medical Science , 75, 1329-1333.https://doi.org/10.1292/jvms.13-018\%.

Liu, G.H., Lin, R.Q., Li, M.W., Liu, W., Liu, Y., Yuan, Z.G., .. Zhu, X.Q. (2011). The complete mitochondrial genomes of three cestode species of Taenia infecting animals and humans. Molecular Biology Reports , 38, 2249-2256.https://doi.org/10.1007/s11033-010-0355-0.

Lymbery A.J. (1998). Combining data from morphological traits and genetic markers to determine transmission cycles in the tape worm,Echinococcus granulosus . Parasitology , 117, 185192.https://doi.org/10.1017/s0031182098002911.

Lymbery, A.J. (2017). Phylogenetic Pattern, Evolutionary Processes and Species Delimitation in the Genus Echinococcus . Advances in Parasitology , 95, 111-145.https://doi.org/10.1016/bs.apar.2016.07.002.

Mathis, A., \& Deplazes, P. (2006). Copro-DNA tests for diagnosis of animal taeniid cestodes. Parasitology International, 55, S87-S90.https://doi.org/10.1016/j.parint.2005.11.012.

Michelet, L., \& Dauga, C. (2012). Molecular evidence of host influences on the evolution and spread of human tapeworms. Biological Reviews of the Cambridge Philosophical Society , 87, 731741.https://doi.org/10.1111/j.1469-185X.2012.00217.x.

Nakao, M., Lavikainen, A., Iwaki, T., Haukisalmi, V., Konyaev, S., Oku, Y., ... Ito, A. (2013). Molecular phylogeny of the genusTaenia (Cestoda: Taeniidae): proposals for the resurrection ofHydatigera Lamarck, 1816 and the creation of a new genus Versteria . International Journal for Parasitology , 43, 427-437.https://doi.org/10.1016/j.ijpara.2012.11.014.

Nakao, M., McManus, D.P., Schantz, P.M., Craig, P.S., \& Ito, A. (2007). A molecular phylogeny of the genus Echinococcus inferred from complete mitochondrial genomes. Parasitology , 134, 713722.https://doi.org/10.1017/S0031182006001934.

Nakao, M., Sako, Y., Yokoyama, N., Fukunaga, M., \& Ito, A. (2000). Mitochondrial genetic code in cestodes.Molecular and Biochemical Parasitology , 111, 415-424.https://doi.org/10.1016/s0166-6851(00)003340 .

Okimoto, R., Macfarlane, J.L., \& Wolstenholme, D.R. (1990). Evidence for the frequent use of TTG as the translation initiation codon of mitochondrial protein genes in the nematodes, Ascaris suum and Caenorhabditis elegans .Nucleic Acids Research , 18, 6113-6118.https://doi.org/10.1093/nar/18.20.6113. 
Paul, R. (2002). Species concepts versus species criteria. Trends in Parasitology , 18, 439440.https://doi.org/10.1016/s1471-4922(02)02319-x.

Posada, D., \& Crandall, K.A. (1998). MODELTEST: testing the model of DNA substitution. Bioinformatics (Oxford, England) , 14, 817-818.https://doi.org/10.1093/bioinformatics/14.9.817 .

Pradhan, N., Sharma, A.N., Sherchan, A.M., Chhetri, S., Shrestha, P., \& Kilpatrick, C.W. (2019). Further assessment of the Genus Neodon and the description of a new species from Nepal. PLoS One, 14, e0219157.https://doi.org/10.1371/journal.pone.021915\%.

Rota-Stabelli, O., Yang, Z., \& Telford, M.J. (2009). MtZoa: A general mitochondrial amino acid substitutions model for animal evolutionary studies. Molecular Phylogenetics and Evolution , 52, 268272.https://doi.org/10.1016/j.ympev.2009.01.011.

Shi, Z.G., Liu, X.D., \& Sha, Y.Y. (2015). Did northern Tibetan Plateau uplift during Pliocene? A modeling test. Journal of Earth Environment , 6, 67-80. (in Chinese)

Singla, L.D., Singla, N., Parshad, V.R., Juyal, P.D., \& Sood, N.K. (2008). Rodents as reservoirs of parasites in India. Integrative Zoology, 3, 21-26.https://doi.org/10.1111/j.1749-4877.2008.00071.x.

Smith, A.T., Badingqiuying, Wilson, M.C., \& Hogan, B.W. (2019). Functional-trait ecology of the plateau pika Ochotona curzoniae in the Qinghai-Tibetan Plateau ecosystem. Integrative Zoology , 14, 87103.https://doi.org/10.1111/1749-4877.12300.

Suzan, G., Marce, E., Giermakowski, J.T., Armien, B., Pascale, J., Mills, J., .. Yates, T. (2008). The effect of habitat fragmentation and species diversity loss on hantavirus prevalence in Panama. Annals of the New York Academy of Sciences , 1149, 80-83.https://doi.org/10.1196/annals.1428.063.

Telford, M.J., Herniou, E.A., Russell, R.B., \& Littlewood, D.T. (2000). Changes in mitochondrial genetic codes as phylogenetic characters: two examples from the flatworms. Proceedings of the National Academy of Sciences of the United States of America , 97, 11359-11364.https://doi.org/10.1073/pnas.97.21.11359 .

Terefe, Y., Hailemariam, Z., Menkir, S., Nakao, M., Lavikainen, A., Haukisalmi, V., ... Ito, A. (2014). Phylogenetic characterisation of Taenia tapeworms in spotted hyenas and reconsideration of the "Out of Africa" hypothesis of Taenia in humans. International Journal for Parasitology , 44, 533541.https://doi.org/10.1016/j.ijpara.2014.03.013.

Turelli, M., Barton, N.H., \& Coyne, J.A. (2001). Theory and speciation. Trends in Ecology \& Evolution , 16, 330-343.https://doi.org/10.1016/s0169-5347(01)02177-2 .

Valdmann, H., Moks, E., \& Talvik, H. (2004). Helminth fauna of Eurasian lynx (Lynx lynx) in Estonia. Journal of Wildlife Diseases , 40, 356-360.https://doi.org/10.7589/0090-3558-40.2.356.

Wang, S., Wang, S., Luo, Y., Xiao, L., Luo, X., Gao, S., .. Cai, X. (2016). Comparative genomics reveals adaptive evolution of Asian tapeworm in switching to a new intermediate host. Nature Communications, 7, 12845.https://doi.org/10.1038/ncomms12845.

Wang, X., Liang, D., Jin, W., Tang, M., Liu, S., \& Zhang, P. (2020). Out of Tibet: Genomic Perspectives on the Evolutionary History of Extant Pikas. Molecular Biology and Evolution , 37, 15771592.https://doi.org/10.1093/molbev/msaa026.

Wang, X., Liu, J., Zuo, Q., Mu, Z., Weng, X., Sun, X., ... Wang, Z. (2018). Echinococcus multilocularis and Echinococcus shiquicus in a small mammal community on the eastern Tibetan Plateau: host species composition, molecular prevalence, and epidemiological implications. Parasites 65 Vectors , 11, 302.https://doi.org/10.1186/s13071-018-2873-x .

Will, K.W., Mishler, B.D., \& Wheeler, Q.D. (2005). The perils of DNA barcoding and the need for integrative taxonomy. Systematic Biology, 54, 844-851.https://doi.org/10.1080/10635150500354878 . 
Wu, Z., Lu, L., Du, J., Yang, L., Ren, X., Liu, B., .. Jin, Q. (2018). Comparative analysis of rodent and small mammal viromes to better understand the wildlife origin of emerging infectious diseases.Microbiome, 6, 178.https://doi.org/10.1186/s40168-018-0554-9.

Xiao, N., Qiu, J., Nakao, M., Li, T., Yang, W., Chen, X., ... Ito, A. (2005). Echinococcus shiquicus n. sp., a taeniid cestode from Tibetan fox and plateau pika in China. International Journal for Parasitology , 35, 693-701.https://doi.org/10.1016/j.ijpara.2005.01.003.

Zhou, L., \& Ma, Y. (2002). Distribution patterns of rodent diversity in arid regions of West China. Biodiversity Science. Chinese Biodiversity , 10, 44-48. (in Chinese)

Table 1. Positions and gene lengths in the mitochondrial genomes of Taenia tianguangfui larva (Tt), $T$. caixuepengi larva (Tc)

\begin{tabular}{|c|c|c|c|c|}
\hline Genes & Positions (length, bp) & Positions (length, bp) & Initiation and termination codons & Initiation and termination $\mathrm{cc}$ \\
\hline & $\mathrm{Tt}$ & Tc & $\mathrm{Tt}$ & $\mathrm{Tc}$ \\
\hline $\operatorname{trn} \mathrm{G}$ & $1-65(65)$ & $1-68(68)$ & & \\
\hline $\cos 3$ & $68-715(648)$ & $72-722(651)$ & ATG/TAG & GTG/TAA \\
\hline $\operatorname{trn} \mathrm{H}$ & $724-792(69)$ & $716-786(71)$ & & \\
\hline$c y t b$ & $796-1863(1068)$ & $790-1857(1068)$ & ATG/TAG & ATG/TAA \\
\hline $\operatorname{nad} 4 \mathrm{~L}$ & $1865-2125(261)$ & $1857-2117(261)$ & ATG/TAG & ATG/TAA \\
\hline nad4 & 2092-3348 (1257) & 2084-3337 (1254) & ATG/TAG & ATG/TAA \\
\hline $\operatorname{trn} \mathrm{Q}$ & $3349-3409(61)$ & $3338-3400(63)$ & & \\
\hline $\operatorname{trn} \mathrm{F}$ & $3409-3472(64)$ & $3400-3463(64)$ & & \\
\hline $\operatorname{trn} \mathrm{M}$ & 3469-3534 (66) & $3461-3524(64)$ & & \\
\hline atp 6 & $3535-4053(519)$ & $3531-4043(513)$ & ATG/TAG & ATT/TAA \\
\hline nad2 & $4058-4930(873)$ & $4045-4917(873)$ & ATG/TAA & ATG/TAG \\
\hline $\operatorname{trn} \mathrm{V}$ & $4947-5011(65)$ & $4922-4983(62)$ & & \\
\hline $\operatorname{trn} \mathrm{A}$ & $5012-5075(64)$ & $4996-5058(63)$ & & \\
\hline $\operatorname{trn} \mathrm{D}$ & $5085-5149(65)$ & $5067-5128(62)$ & & \\
\hline$n a d 1$ & $5154-6047(894)$ & $5134-6030(897)$ & ATG/TAA & ATG/TAA \\
\hline $\operatorname{trn} \mathrm{N}$ & 6064-6131 (68) & 6044-6109 (66) & & \\
\hline $\operatorname{trn} \mathrm{P}$ & 6141-6205 (65) & $6118-6180(63)$ & & \\
\hline $\operatorname{trn} \mathrm{I}$ & $6205-6267(63)$ & $6181-6244(64)$ & & \\
\hline $\operatorname{trn} \mathrm{K}$ & 6273-6338 (66) & 6246-6309 (64) & & \\
\hline nad3 & $6342-6689(348)$ & 6310-6657 (348) & ATG/TAA & GTG/TAA \\
\hline $\operatorname{trn} \mathrm{S}$ & 6689-6747 (59) & $6656-6716(61)$ & & \\
\hline $\operatorname{trn} \mathrm{W}$ & $6755-6820(66)$ & 6716-6778 (63) & & \\
\hline $\operatorname{cox} 1$ & $6824-8443(1620)$ & $6782-8401(1620)$ & ATG/TAA & ATG/TAA \\
\hline $\operatorname{trn} \mathrm{T}$ & 8429-8495 (67) & $8387-8451(65)$ & & \\
\hline$r r n \mathrm{~L}$ & 8496-9468 (973) & 8452-9412 (961) & & \\
\hline $\operatorname{trn} \mathrm{C}$ & $9469-9529(61)$ & $9418-9475(58)$ & & \\
\hline $\operatorname{rrn} \mathrm{S}$ & $9530-10266(737)$ & $9476-10200(725)$ & & \\
\hline $\cos 2$ & $10267-10844(578)$ & $10201-10785(585)$ & ATG/TAA & ATG/TAA \\
\hline $\operatorname{trn} \mathrm{E}$ & $10853-10920(68)$ & $10787-10853(67)$ & & \\
\hline $\operatorname{nad} 6$ & $10923-11375(453)$ & $10855-11307(453)$ & ATG/TAA & ATG/TAG \\
\hline $\operatorname{trn} \mathrm{Y}$ & $11379-11441(63)$ & $11314-11376(63)$ & & \\
\hline SNR & $11442-11508(67)$ & $11377-11441(65)$ & & \\
\hline $\operatorname{trn} \mathrm{L}$ & $11509-11574(66)$ & $11443-11512(70)$ & & \\
\hline $\operatorname{trn} \mathrm{S}$ & $11604-11661$ (58) & $11550-11609(60)$ & & \\
\hline $\operatorname{trn} \mathrm{L}$ & $11673-11738(66)$ & $11612-11680(69)$ & & \\
\hline $\operatorname{trn} \mathrm{R}$ & $11744-11802(59)$ & $11680-11734(55)$ & & \\
\hline nad5 & $11803-13371(1569)$ & $11729-13303(1575)$ & GTG/TAA & ATG/TAA \\
\hline
\end{tabular}




\begin{tabular}{lllll}
\hline Genes & Positions (length, bp) & Positions (length, bp) & Initiation and termination codons & Initiation and termination co \\
\hline LNR & $13372-13522(151)$ & $13304-13747(444)$ & & \\
\hline
\end{tabular}

\section{Figure legends}

Figure 1. The diagram of complete mitochondrial genome of Taenia tianguangfui (A) and $T$. caixuepengi (B). The protein-encoding genes are depicted in plum, the tRNAs are depicted in green, the rRNAs are depicted in light green and the non-coding mitochondrial regions (NCRs including LNR and SNR) are depicted in gray. The inferred gene boundaries of them are shown in Table 1.

Figure 2. The phylogenetic relationship of Taenia tianguangfui and T. caixuepengi, with other 32 tapeworm species inferred from a Bayesian method based on the concatenated amino acid (A) and CDS alignments (B) of mitochondrial 12 protein-encoding genes. The species' name corresponding to the GenBank ID is given in the Supplementary Table S2. The Echinococcus spp. are depicted in red, the Taenia spp. are depicted in blue, the Hydatigeraspp. are depicted in yellow and the only one Versteria species, Versteria mustelae, is depicted in green. The Schistosoma japonicum depicted in black was chosen as outgroup.

Figure 3. Divergence times construction for Taenia tianguangfui and T. caixuepengi based on the concatenated CDS alignments of mitochondrial 12 protein-encoding genes. The number at the node represents the divergence time between two lineages. The blue bar represents interval of $95 \%$ highest probability density, and the number in brackets represents the detailed time interval of $95 \%$ highest probability density of divergence time between two lineages. A time scale shows the extent of the Miocene, Pliocene and Pleistocene period.

Supplementary figure legends

Supplementary Figure S1. The larvae of Taenia caixuepengiand T. tianguangfui were found in Plateau pikas (Ochotona curzoniae) and Qinghai voles (Neodon fuscus), respectively. The banded larvae of $T$. caixuepengi in the plate (A) were picked out from the enterocoelia of Plateau pikas (B), and the granular larvae of $T$. tianguangfui in the plate $(\mathrm{C})$ were picked out from the enterocoelia and chest of Qinghai voles (D). The blue arrow indicates the parasitic larvae.

Supplementary Figure S2. Divergence times construction for Neodon fuscus based on the concatenated CDS alignments of mitochondrial 13 protein-encoding genes. The number at the node represents the divergence time between two lineages. The blue bar represents interval of $95 \%$ highest probability density, and the number in brackets represents the detailed time interval of $95 \%$ highest probability density of divergence time between two lineages. A time scale shows the extent of the Miocene, Pliocene and Pleistocene period. 

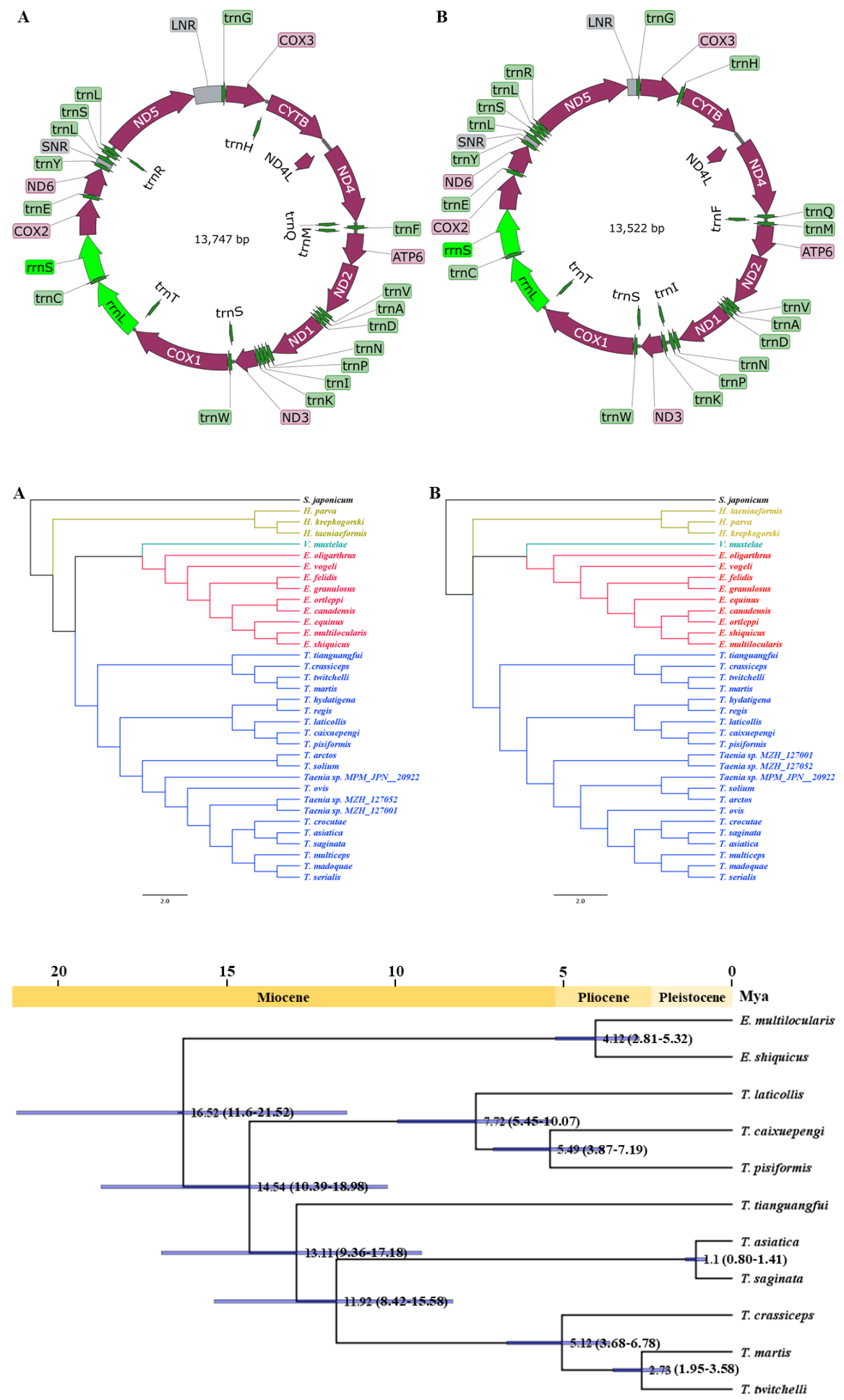

2.0 